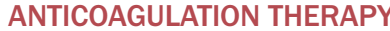

\section{Apixaban indications set to AMPLIFY?}

The anticoagulant apixaban is easier to administer than warfarin, and has garnered much attention in the settings of atrial fibrillation and venous thromboembolism (VTE). The AMPLIFY-EXT results, published in late 2012, showed that apixaban might be useful as an extended therapy for VTE. The AMPLIFY investigators have now reported on the safety and efficacy of apixaban as a first-line therapy for the treatment of patients with acute VTE.

In the randomized, double-blind study, oral administration of apixaban was compared with conventional first-line treatment-subcutaneous injection of enoxaparin, followed by warfarin - in nearly 5,400 patients with pulmonary embolism, deep-vein thrombosis, or both. Individuals enrolled in the trial received either a $10 \mathrm{mg}$ dose of apixaban twice daily for 7 days and then $5 \mathrm{mg}$ twice daily for 6 months $(n=2,691)$, or $1 \mathrm{mg}$ enoxaparin per $\mathrm{kg}$ of body weight every $12 \mathrm{~h}$ over at least 5 days, with concomitant warfarin, and then warfarin alone for 6 months $(n=2,704)$.
Apixaban was as effective as conventional therapy in preventing recurrent symptomatic VTE or VTErelated death, the primary efficacy outcome (2.3\% vs $2.7 \%$; RR 0.84, 95\% CI $0.60-1.18)$. More notably, the incidence of major bleeding, the primary safety outcome, was significantly lower with apixaban $(0.6 \%$ vs $1.8 \%$ with conventional therapy; RR 0.31, 95\% CI 0.17-0.55). Incidents of major or clinically relevant nonmajor bleeding were also significantly reduced in the apixaban group $(4.3 \%$ vs 9.7\%; RR 0.44, 95\% CI 0.36-0.55).

The AMPLIFY investigators conclude that apixaban is a safe, easily administered alternative to conventional therapies for the initial and long-term treatment of acute, symptomatic VTE.

Tim Geach

Original article Agnelli, G. et al. Oral apixaban for the treatment of acute venous thromboembolism. N. Engl. J. Med. doi:10.1056/NEJMoa1302507 